\title{
"Implementación del Sistema de Gestión de Servicio en el proceso de certificación de productos software y hardware en la Subgerencia de Aseguramiento de Entidades Financieras"
}

\section{"Implementation of the Service Management System in the process of certification of software and hardware products in the Sub-Management of Financial Entities Assurance"}

\section{"Implementação do Sistema de Gerenciamento de Serviços no processo de certificação de produtos de software e hardware na Sub-Gestão da Garantia de Entidades Financeiras"}

\author{
Jorge Martin Figueroa Revilla ${ }^{18}$; Ana Doris Magdalena Barrera Loza ${ }^{19}$; Andrea Gianella Churrango
} Romero $^{20}$; Yannira Del Pilar Mosquera Reyes ${ }^{21}$.

\begin{abstract}
RESUMEN:
El motivo del presente artículo es dar a conocer la experiencia de la Implementación de un sistema de gestión de servicios en el proceso de certificación de los productos software y hardware en la Subgerencia de Aseguramiento de la Calidad de las Entidades Financieras alineadas a las mejores prácticas de la Norma UNE-ISO(IEC_27001=2014, y UNE-ISO(IEC_27002=2014 para la ejecución de las peticiones de servicios o testing que se realiza en un ambiente previo o de pre producción; para ello el sistema de gestión, ha utilizado los procesos que fueron requeridas por la norma internacional; Las Gerencias de Informática, deben definir un Plan estratégico de tecnología alineadas al Plan Estratégico Institucional; Así mismo generar un nuevo Plan Estratégico de Tecnología PETI. La Gerencia de Informática de cada Entidad financiera, en su enfoque de liderazgo en el uso de las Tecnologías de Información y sobre todo orientado a liderar proyectos de transformación Digital; requieren contar con un área que le permita el aseguramiento de la calidad en las soluciones que ingresan a producción, es por ello que, para conseguir la certificación internacional, requieren consolidar dicho esfuerzo. Para que cuando obtengan la ratificación y ampliación de la certificación internacional del sistema de Gestión del proceso de certificación de productos Software y Hardware, se van a fortalecer con una madurez necesaria para los cambios y proyectos que se va a implementar en las entidades financieras. Como resultado del especial esfuerzo de cada entidad financiera, podrán llegar a conseguir la Re-Certificación en la ISO (IEC_27001=2014.
\end{abstract}

Palabras clave: Mejora de la productividad, Sistema de Gestión de Servicios, UNE-ISO (IEC_27001=2014), Niveles de Acuerdos de Servicios, Peticiones de Servicios Rechazada y/o Devueltos.

\footnotetext{
ABSTRACT:

The reason for this article is to present the experience of the Implementation of a service management system in the process of certification of software and hardware products in the Sub-Management of Quality Assurance of Financial Institutions aligned to the best practices of the UNE-ISO Standard (IEC_27001 $=2014$, and UNE-ISO (IEC_27002 $=2014$ for the execution of service or testing requests that are carried out in a pre-production or pre-production environment; for this the management system has used the processes that were required by the international standard; IT Managements must define a Strategic Technology Plan aligned to the Institutional Strategic Plan; Likewise, generate a new PETI

18 Autor: Jorge Martin Figueroa Revilla, Intecon Peru S.A.C., Universidad San Martin de Porres, Universidad Nacional José Faustino Sánchez Carrión; Facultad de Ingeniería Industrial, Sistemas e Informática, Email: jfigueroar@usmp.pe y mfigueroa@inteconperu.com. Orcid: /0000-0003-0884-2571

${ }^{19}$ Coautor: Ana Doris Magdalena Barrera Loza, Universidad Nacional José Faustino Sánchez Carrión; Facultad de Ingeniería Industrial, Sistemas e Informática. Email: anadobar@ hotmail.com, Orcid: 0000-0001-8296-6519

${ }^{20}$ Coautor: Andrea Gianella Churrango Romero, Intecon Peru SAC. analista de TI Junior, Email: agcr.1423@ hotmail.com

${ }^{21}$ Coautor: Yannira Del Pilar Mosquera Reyes, Intecon Peru SAC. analista de TI Junior, Email: reyesyannira@gmail.com
} 
Technology Strategic Plan. The Information Technology Management of each Financial Entity, in its leadership approach in the use of Information Technology and especially oriented to lead Digital transformation projects; They require an area that allows quality assurance in the solutions that enter production, which is why, to achieve international certification, they need to consolidate this effort. So that when they obtain the ratification and extension of the international certification of the Management System of the Software and Hardware product certification process, they will be strengthened with a necessary maturity for the changes and projects that will be implemented in the financial entities. As a result of the special effort of each financial institution, they will be able to achieve the Re-Certification in the ISO (IEC_27001 = 2014 .

Keywords: Productivity improvement, Service Management System, UNE-ISO (IEC_27001 = 2014, Service Agreement Levels, Rejected and / or Returned Service Requests.

\section{RESUMO:}

O motivo deste artigo é apresentar a experiência da Implementação de um sistema de gerenciamento de serviços no processo de certificação de produtos de software e hardware na Sub-Gestão da Garantia da Qualidade das Instituições Financeiras, alinhada às melhores práticas de a norma UNE-ISO (IEC_27001 = 2014 e UNE-ISO (IEC_27002 = 2014 para a execução de solicitações de serviço ou teste que são realizadas em um ambiente de pré-produção ou pré-produção; para isso, o sistema de gerenciamento usou o processos exigidos pelo padrão internacional; Gerências de TI devem definir um Plano Estratégico de Tecnologia alinhado ao Plano Estratégico Institucional; Da mesma forma, gerar um novo Plano Estratégico de Tecnologia PETI. A Gestão da Tecnologia da Informação de cada Entidade Financeira, em sua abordagem de liderança no uso da Tecnologia da Informação e especialmente orientada para liderar projetos de transformação Digital; Eles exigem uma área que permita garantia de qualidade nas soluções que entram em produção e, por isso, para obter a certificação internacional, eles precisam consolidar esse esforço. Para que, ao obterem a ratificação e extensão da certificação internacional do Sistema de Gerenciamento do processo de certificação de produtos de Software e Hardware, sejam fortalecidos com a maturidade necessária para as mudanças e projetos que serão implementados nas entidades financeiras. Como resultado do esforço especial de cada instituição financeira, elas poderão obter a recertificação na ISO (IEC_27001 $=2014$.

Palavras-chave: Melhoria da produtividade, sistema de gerenciamento de serviços, UNE-ISO (IEC_27001 = 2014), níveis de contrato de serviço, solicitações de serviço rejeitadas e / ou devolvidas.

\section{Introducción:}

Una entidad financiera es cualquier entidad o agrupación que tiene como objetivo y fin ofrecer servicios de carácter financiero y que van desde la simple intermediación y asesoramiento al mercado de los seguros o créditos bancarios.

Las instituciones financieras como bancos, cooperativas de crédito, corredores de bolsas, compañías de seguros y finanzas, a menudo tienen un plan de negocios con una lista de metas y objetivos. Estos objetivos son un conjunto de normas o metas, por las cuales la institución en su totalidad y cada empleado trabajan todos los días. Los objetivos pueden ser externos y beneficiar a los clientes, pero también pueden tener beneficios externos y crear una marca de la institución financiera.

Las entidades financieras están encaminadas a crecer con nuevos productos y servicios, es por ello que debe estar orientado hacia la transformación digital, la Banca Digital y consolidar su presencia financiera como única oferta bancaria. La Subgerencia Aseguramiento de la Calidad de Soluciones de cada entidad se encarga de asegurar que las nuevas soluciones en Tecnologías o los cambios en aquellos existentes pasen a producción o se liberen en el ambiente productivo con la calidad e integridad necesaria para el correcto funcionamiento de los servicios financieros que brinda a todo el sector, así como las operaciones Bancarias para que se realicen con la integridad, exactitud requeridas. Las entidades financieras requieren de mecanismos que les aseguren que las soluciones tecnológicas que liberan a sus ambientes productivos dispongan de los controles requeridos, es por ello que requieren contar con dichos mecanismos de aseguramiento, de protocolos y procedimientos eficaces. Ya que el servicio de certificación del producto software y hardware, no puede permitirse ningún error o por lo menos los riesgos deben ser mininos y controlados. 
Existe un antes y un después, es decir antes de la Implementación del Sistema de Gestión y un después. En el cual, la mejora de la productividad y el orientarse en el cumplimiento de niveles de servicios ahora con dos servicios que brinda la Subgerencia de Aseguramiento de la Calidad como es la certificación de productos Software y Hardware, sus protocolos, procedimientos y controles tienen que ser completos, consolidados y eficaces. Es por ello que la falta de contar con todos los protocolos, procedimientos y controles genera que el servicio no cumpla con su objetivo principal de asegurador que las soluciones de tecnología se liberen con la confiabilidad requerida.

Obteniendo la certificación internacional ayudara a la Entidad Financiera, ya que ahora las peticiones de servicios no demoraran más tiempo en ser atendía. Por estas razones, se implementa el sistema de gestión de servicio para dar fortalecer el proceso de certificación tanto en los servicios de productos software como hardware.

\section{Materiales y Métodos}

En esta investigación se utilizó el método Deductivo, se utilizó para sacar conclusiones de los antecedentes de la investigación, de los autores de teorías sobre la implementación de los sistemas de gestión o de la ISO 20000. La presente investigación desarrollada es de tipo aplicada o tecnológica orientada a problemas actuales, concretos e identificables en el proceso de certificación de productos software en la Sub Gerencia de Aseguramiento de calidad del Banco de la Nación.

El diseño de la investigación es Experimental, de nivel explicativa, de tipo Pre-experimental, porque va más allá de la descripción de conceptos y busca el porqué de los hechos mediante el establecimiento de relaciones causa-efecto.

La población está conformada por todas las Peticiones de Servicios que se formularon a la Sub Gerencia de Aseguramiento de la Calidad durante los años 2013, 2014, y 2015 para un antes y para el después los años 2016, 2017, 2018 y para calcular el tamaño de una muestra, se utilizó la técnica de muestreo probabilístico.

Tabla 1

No existía el Sistema de Gestión

\begin{tabular}{lcc}
\hline Años & $\begin{array}{c}\text { Total } \\
\text { Población }\end{array}$ & Muestra \\
\hline Año 2013 & 701 & 26 \\
Año 2014 & 580 & 21 \\
Año 2015 & 730 & 27 \\
\hline
\end{tabular}

Tabla 2

Después de Implementar el Sistema de Gestión de Servicios

\begin{tabular}{l|cc}
\hline Años & $\begin{array}{c}\text { Total } \\
\text { Población }\end{array}$ & Muestra \\
\hline Año 2016 & 967 & 36 \\
Año 2017 & 963 & 36 \\
Año 2018 & 1020 & 38 \\
\hline
\end{tabular}

Para la investigación se utilizó los siguientes instrumentos:

- IBM Rational ClearQuest

- ITOP

- Requisite Pro

- Host On Demand y IBM Personal communication

- Las dos son herramientas IBM que permiten la emulación de un terminal virtual con acceso a los sistemas HOST.

-WebSphere

- Websphere

-WAS, WebSphere Application Server

-WebSphere MQ

- WebSphere Broker 
Para el procedimiento, con la información obtenida mediante la herramienta Rational y los años de recolección desde el 2011 hasta el 2015 de todas las prestaciones de servicios y después de implementar el Sistema de Gestión de la ISO 20000, se procedió a determinar el valor estadístico, para lo cual tomamos la información de los años 2015,2014,2013 anteriores y después del año $2016,2017,2018$.

\section{Resultados}

Los resultados deben ser presentados en una secuencia lógica en el texto, tablas y figuras, se debe evitar la presentación repetida de los mismos datos en diferentes formas. Al describir los resultados de los experimentos de los autores, esto debe ser escrito en tiempo pasado. Los resultados deben ser explicados, pero en gran parte sin hacer referencia a la literatura.

\section{Discusión}

Se muestra la implementación del sistema de Gestión de servicio en el proceso de Certificación de software en la Sección Calidad de Soluciones de las Entidades Financieras, lo cual mejora la productividad, permite el cumplimiento de los niveles de servicios, reduce las peticiones rechazados a la división de desarrollo de sistemas por parte de la sección calidad de soluciones y finalmente reduce las peticiones de servicios devuelta a la sección calidad de soluciones por parte de la sección operaciones y plataforma de equipos.

Así mismo, se ve los resultados en otras actividades administrativas y el seguimiento de las peticiones después de haber sido desplegadas en el ambiente de producción.

\section{Conclusión}

Con la implementación de un Sistema de Gestión de Servicio se mejora sustancialmente la eficiencia en el proceso de certificación de productos software, ya que se tiene control de los procesos, recursos y utilización de los niveles de servicios apropiadamente. Para ello a continuación plasmamos las principales conclusiones de nuestra investigación:

Se pudo comprobar que con la implementación de un sistema de gestión de servicio basadas en la Norma ISO 20000 para mejorar el proceso de certificación de los productos software en las entidades financieras, ha incrementado en un $99 \%$ la efectividad en el servicio que brinda a las áreas internas de cada entidad.

Del mismo modo la implementación de un sistema de gestión de servicio basadas en la Norma ISO 20000 permitió mejorar en un 98\% la atención de las peticiones de servicios en el proceso de certificación de los productos software en las entidades financieras.

Así mismo con la implementación de un sistema de gestión de servicio basadas en la Norma ISO 20000 redujo en un $98 \%$ las peticiones de servicios rechazados a la división de desarrollo de sistemas en el proceso de certificación de los productos software en las entidades financieras.

Finalmente, con la implementación de un sistema de gestión de servicio basadas en la Norma ISO 20000 redujo en un 98\% las peticiones de servicios devueltas por la Sección Operaciones de Equipos y Control de Plataforma en el proceso de certificación de los productos software en las entidades financieras.

\section{Referencias bibliográficas}

AENOR. (2016). Ficha de Certificado de la Empresa. Recuperado de http://www.aenor.es/aenor/certificacion/buscador/fichacertificado.asp?empresaAenor=PER\& codigo $=120794 \#$.VAs0Kha0emx

AENOR, Asociación Española de Normalización y Certificación. (2011). Tecnología de la información Gestión del Servicio Parte 1: Requisitos del Sistema de Gestión del Servicio (SGS). AENOR.

AGENCIA DE NOTICIAS ORBITA. (2016). Cosapi Data obtiene certificaciones ISO/IEC 20000 e ISO/IEC 27001, gracias a la consultoria de PMC® | Agencia Orbita. Recuperado de http://agenciaorbita.org/cosapi-data-obtiene-certificaciones-isoiec-20000-e-isoiec-27001gracias-a-la-consultoria-de-pmc/

BAUSET, M. y Rodones, M. (2013). Gestión De Los Servicios De Tecnologías De La Información: Modelo De Aporte...: EBSCOhost. En Gestión de los servicios de tecnologías 
de la información (pp. 54-61). Recuperado de

https://web.a.ebscohost.com/ehost/detail/detail?vid=4\&sid=5490c542-cc22-4caf-8754-

c781a2398417\%40sessionmgr4003\&hid=4214\&bdata=Jmxhbmc9ZXMmc210ZT1laG9zdC1 $\underline{\mathrm{saXZl} \# \mathrm{db}=\mathrm{bth} \& \mathrm{AN}=92774922}$ 